\title{
Case reports of iatrogenic vascular injury in the trauma field: what is the same and what is different?
}

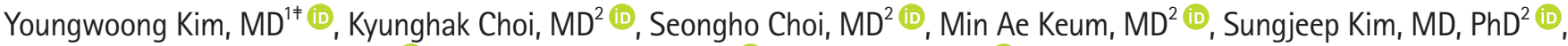

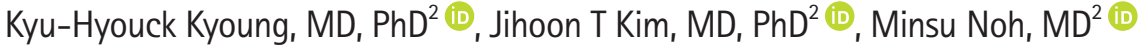 \\ 1 Department of Thoracic and Cardiovascular Surgery, Ulsan University Hospital, University of Ulsan College of Medicine, Ulsan, Korea \\ ${ }^{2}$ Department of Trauma Surgery, Ulsan University Hospital, University of Ulsan College of Medicine, Ulsan, Korea
}

Received: September 15, 2021

Revised: December 10, 2021

Accepted: December 16, 2021

Correspondence to

Minsu Noh, MD

Department of Trauma Surgery, Ulsan

University Hospital, University of Ulsan

College of Medicine, 877

Bangeojinsunhwando- ro, Dong-gu,

Ulsan 44033, Korea

Tel: +82-52-250-7118

E-mail: leominsunoh@uuh.ulsan.kr

${ }^{\ddagger}$ Current affiliation: Department of Thoracic and Cardiovascular Surgery, Gangnam Severance Hospital, Yonsei University College of Medicine, Seoul, Korea
Iatrogenic vascular injury (IVI) can occur with any technique or type of surgery performed around a blood vessel. Patients with severe trauma are at risk of IVI. In this study, we describe our experiences of IVI in the trauma field. We reviewed five patients who were diagnosed with an IVI and received either surgical or endovascular treatment. Of the five patients, one had an arterial injury, three had venous injuries, and one had an arteriovenous fistula, a form of combined arterial and venous injuries. Of the five patients, four had undergone orthopedic surgery. The IVIs of three patients were immediately identified in the operating room and simultaneous vascular repair was performed. The remaining one patient underwent additional surgery for occlusion related to entrapment of the superficial femoral artery by a surgical wire used during orthopedic surgery. Complications presumably related to the IVI were identified in two patients. IVI in trauma patients can be successfully managed, but significant morbidity can occur. If an IVI is suspected, immediate evaluation and management are required.

Keywords: Vascular injury; Iatrogenic injury; Complications; Case report

\section{INTRODUCTION}

Iatrogenic vascular injury (IVI) can occur in various medical and surgical settings. As endovascular procedures have been widely used to diagnose and treat patients, the frequency of IVI is reported to have increased [1,2]. Cases of IVI occur during surgery and other situations that require vascular access [3]. However, IVI in the trauma field has not been characterized and investigated. An early multidisciplinary approach and emergency surgery are required for patients with severe trauma to assess the extent of organ damage and the complexity of the injury. Multiple vascular access sites are often required to respond to early shock and/or for longterm intensive care unit (ICU) treatment and monitoring [4]. IVI can cause secondary damage in trauma patients by creating an additional cycle of bleeding [3]. This study describes our experiences with IVIs during surgical or endovascular treatments at a single regional trauma center. 


\section{CASE REPORTS}

Clinical characteristics of five patients treated for IVI, and diagnostic and treatment strategies and clinical outcomes are summarized in Tables 1, 2. Informed consent for publication of the research details and clinical images was obtained from all individual participants included in this study.

\section{Case 1}

A 71-year-old male patient who was undergoing regular hemodialysis through the right native forearm arteriovenous fistula (AVF) for underlying end-stage renal disease presented to the emergency room (ER) complaining of pain in the right shoulder due to trauma. The patient was diagnosed with a surgical neck fracture of the right humerus on examination, and open reduction and internal fixation (ORIF) was performed on the third day of admission. During the operation, bleeding occurred due to damage to the distal cephalic vein. In order to maintain the native AVF, the trauma vascular surgeon was contacted, and primary lateral repair was performed simultaneously. After the operation, hemodialysis was attempted through the AVF according to the regular dialysis schedule and hemodialysis proceeded without any problems.

\section{Case 2}

A 37-year-old male patient without underlying disease presented to the ER complaining of damage caused when his left lower extremity (LE) was caught between a forklift and a railing. The patient was diagnosed with a left tibiofibular open fracture with a popliteal artery injury, and emergency surgery was performed. According to the surgical findings, popliteal artery transection was confirmed, and segmental interposition using the reversed greater saphenous vein was performed. After vascular surgery, the orthopedic surgery team took over the operation and performed ORIF. After surgery, there was sudden bleeding at the site of vascular surgery. As a result of revision, the popliteal vein injury, which was intact, was confirmed. The popliteal vein was transected, and proximal and distal ligations were performed in a situation where approximation was not possible. The patient had substantial muscle mass and the ischemia time was approximately 4 hours. The calf was firm to palpation at the time of revision and prophylactic fasciotomy was performed to prevent progression to compartment syndrome. After 12 days, external fixation conversion was performed, and 16 weeks of wound care were required in collaboration with a plastic surgeon.

\section{Case 3}

A 30-year-old male patient, complaining of abdominal pain following a traffic accident (TA), presented to the ER. The patient was admitted to the ICU due to the possibility of damage to the abdominal wall and internal organs and was clinically followed. A central line insertion was attempted through the right internal

Table 1. Clinical characteristics of five patients treated for IVI

\begin{tabular}{|c|c|c|c|c|c|c|c|}
\hline Patient & $\begin{array}{l}\text { Age } \\
(\mathrm{yr})\end{array}$ & Sex & Diagnosis related with IVI & Site & Vessel related with IVI & Pathology & ISS \\
\hline 1 & 71 & Male & Humerus fracture & Right & Cephalic vein (AVF for dialysis) & Bleeding & 5 \\
\hline 2 & 37 & Male & $\begin{array}{l}\text { Tibio-fibula fracture popliteal artery } \\
\text { transection }\end{array}$ & Left & Popliteal vein & Bleeding & 13 \\
\hline 3 & 30 & Male & S-colon perforation & Right & Subclavian artery, internal jugular vein & AVF & 5 \\
\hline 4 & 75 & Female & Femur fracture & Right & Superficial femoral artery & Occlusion (entrapment) & 25 \\
\hline 5 & 83 & Female & Pelvic fracture & Left & External iliac vein & Bleeding & 22 \\
\hline
\end{tabular}

IVI, iatrogenic vascular injury; ISS, Injury Severity Score; AVF, arteriovenous fistula.

Table 2. Diagnostic and treatment strategies and clinical outcomes in five patients with IVI

\begin{tabular}{llllrr}
\hline Patient & Diagnostic modality (interval, day) & Treatment & Complication & ICU stay (day) & Hospital stay (day) \\
\hline 1 & Operative finding (0) & Lateral repair & None & 0 & 3 \\
2 & Operative finding (0) & Ligation & Calf swelling & 7 & 120 \\
3 & CT angiography (6) & Angio-embolization & None & 0 & 29 \\
4 & CT angiography (14) & Graft interposition & Foot drop & 33 & 133 \\
5 & Operative finding (0) & Segmental R\&A & None & 17 & 33 \\
\hline
\end{tabular}

IVI, iatrogenic vascular injury; ICU, intensive care unit; CT, computed tomography; R\&A, resection and anastomosis. 
jugular vein (IJV) during resuscitation in the ER. An arterial puncture occurred during the attempt to insert a central venous catheter, and the procedure was stopped. No additional complications were observed at the site. On the second day of hospitalization, the patient complained of abrupt abdominal pain, suggesting peritoneal irritation, and emergency exploratory laparotomy was performed. According to the surgical findings, ischemic sigmoid colon perforation caused by damage to the sigmoid mesocolon was diagnosed, and a Hartmann procedure was performed. On the day after surgery, thrill and bruit were observed in the patient's right neck, and arterial flow in the jugular vein was confirmed by bedside Doppler ultrasonography. An additional examination was planned for a suspected iatrogenic AVF. A subclavian artery to IJV AVF was confirmed by computed tomography angiography of the neck (Fig. 1A). Conventional angiography was performed to control the fistula tract (Fig. 1B). After selecting the tract, multiple coil embolization was performed. The final angiography after the procedure confirmed that there was no arterial flow into the IJV (Fig. 1C). There were no additional vascular complications on subsequent follow-up.

\section{Case 4}

A 75-year-old female patient who was diagnosed with a right femur fracture, multiple pelvic fractures, bladder injury, and a fifth lumbar vertebral body fracture due to a pedestrian TA was transferred from a local hospital to the trauma center through the ER for further evaluation and treatment. The patient was admitted to the ICU, and closed reduction and internal fixation for the femur fracture and surgery for the pelvic fractures were performed on the seventh and 11th days after admission, respectively. Postoperatively, there were findings of suspected ischemic injury in the right LE. The initial findings were suspicious for underlying vascular disease, but additional examinations were planned according to the progressive findings. On computed tomography angiography of the LE, segmental arterial occlusion was found at the site of the femur fracture, and findings adjacent to the orthopedic surgical site were observed (Fig. 2A). Therefore, additional surgery was performed, which confirmed that the superficial femoral artery was entrapped by two of the surgical wires used during closed reduction and internal fixation (Fig. 2B). The surgical wires were removed and segmental interposition using a prosthetic graft for the injured superficial femoral artery was performed. There were no additional vascular complications, although the patient did show foot drop during the recovery process, a possible sequela of ischemic peripheral nerve damage.

\section{Case 5}

An 83-year-old female patient presented to the ER complaining of multiple injuries caused by a pedestrian TA. The patient was admitted to the ICU after being diagnosed with a seventh cervical vertebral body fracture, multiple rib fractures, sternum fracture, multiple pelvic fractures, left tibiofibular fracture, and left shoulder fracture. On the 4 th day of admission, surgery was performed for her sternum fracture, multiple pelvic bone fractures, and left tibiofibular fracture. Sudden bleeding was observed at the time of placement of a drainage tube for surgical bed drainage during surgery for the pelvic fracture. A left external iliac
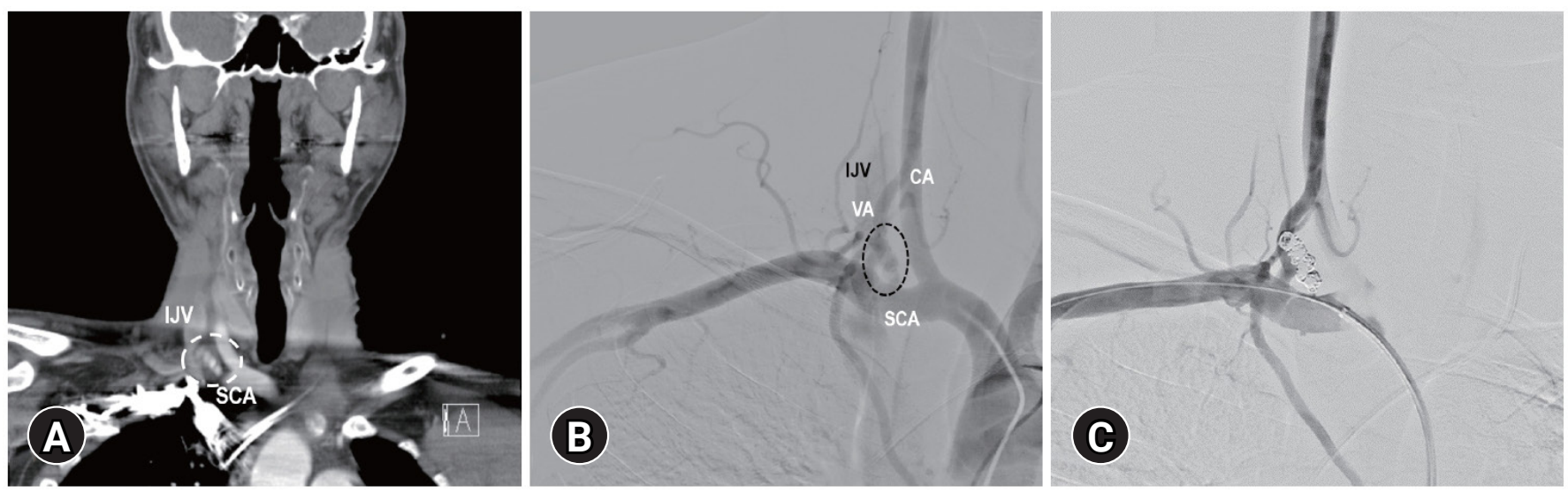

Fig. 1. Arteriovenous fistula between internal jugular vein (IJV) and subclavian artery (SCA) in case 3. (A) Findings of computed tomography angiography. Fistula tract (dotted circle) observed between IJV and SCA. (B) Findings of conventional angiography. Fistula tract (dotted circle) and IJV shown with contrast on angiography. (C) Arteriography after embolization with multiple coils confirmed that there was no contrast of the IJV. CA, carotid artery; VA, vertebral artery. 

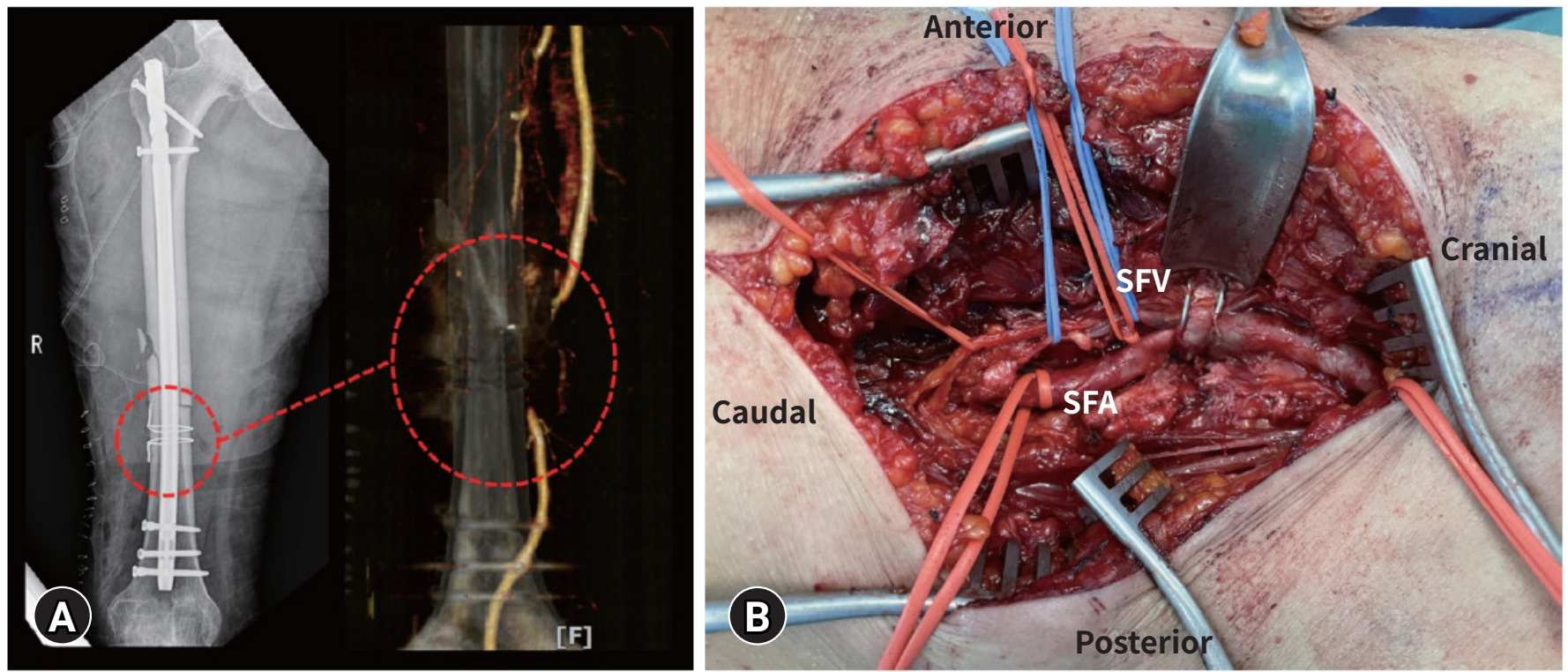

Fig. 2. Superficial femoral artery (SFA) entrapment by two surgical wires used during surgery for femur fracture in case 4. (A) Findings of $\mathrm{X}$-ray and computed tomography angiography. The occluded segment of SFA on computed tomography angiography corresponds to the area of surgical wires applied on X-ray (dotted circle). (B) Operative findings. SFV, superficial femoral vein.

vein injury was suspected, and the trauma vascular surgeon was contacted. On exploration of the bleeding site, anteroposterior penetration of the left external iliac vein was confirmed. Segmental resection and endto-end anastomosis were performed for the injured vein. The patient recovered without additional vascular complications.

\section{DISCUSSION}

IVI is a generic term for vascular damage that occurs during medical diagnostic examination and treatment, and may appear as arterial, venous, or combined arteriovenous damage [5]. The injury can present as bleeding, hematoma, intimal damage, occlusion, or an AVF, and treatment is based on the anatomy, physiological characteristics, and target organ perfusion of the damaged blood vessel [6]. Of the five cases in our institution, there was one case of arterial injury, three cases of venous injury, and one case of AVF in which both artery and vein were damaged. Bleeding occurred in all venous injuries, and the one arterial case was occlusion by entrapment. There were three cases of pseudoaneurysm of the deep femoral artery, suspected of being IVI, but the possibility of damage at the time of trauma due to fracture could not be completely ruled out, so they were excluded.

As endovascular treatments have become more common and the target applications have become more diversified, the inci- dence of IVI related to endovascular treatment has increased $[1,2,6]$. Among trauma patients at our institution, three cases of IVI occurred during an endovascular procedure and all were related to puncture site bleeding and hematoma. Two cases improved with conservative treatment, and one case was treated with a simultaneous endovascular procedure.

As the role of endovascular procedures expands in the trauma field, reports of IVI should be studied and fully considered [7]. However, IVI in the trauma field must also be considered in terms of trauma dealing with multi-organ complex injuries and critically ill patients, multidisciplinary emergency surgery, and specialties related to long-term intensive care [8-10].

Traditional vascular surgical procedures such as primary repair, ligation, and thrombectomy with angioplasty, as well as more recent interventions utilizing endovascular procedures, are widely performed today $[11,12]$. Early diagnosis and treatment, as well as early intervention by a vascular specialist, are essential [4].

As shown in the cases of this study, early detection of damage and simultaneous intervention can result in a stable course, without additional surgery or procedures. Conversely, if the radiological diagnosis of IVI is made postoperatively, after symptoms develop, delayed diagnosis and treatment may result in additional procedures or surgery. In this study, in cases 3 and 4 with delayed diagnosis of IVI, the occurrence of complications related to delayed diagnosis was not observed. Delayed diagnosis of IVI 
which may result in prolonged bleeding or ischemia increases the risk.

In terms of early diagnosis and treatment approaches, the treatment strategy for IVI as well as traumatic vascular injury is solid. When considering the characteristics of patients with severe trauma during early multidisciplinary emergency surgery and procedures, it should be possible to distinguish an IVI from a natural traumatic vascular injury.

IVI in trauma patients can be successfully managed, but significant morbidity can occur. If IVI is suspected, immediate evaluation and appropriate management are required.

\section{NOTES}

\section{Ethical statement}

Informed consent for publication of the research details and clinical images was obtained from all individual participants included in this study.

\section{Conflicts of interest}

The authors have no conflicts of interest to declare.

\section{Funding}

None.

\section{Author contributions}

Conceptualization: all authors; Data curation: YK, MN; Methodology: YK, KC, MN; Project administration: SC, MN; Visualization: SC, MAK, MN; Writing-original draft: YK, KC, MN; Writing-review \& editing: YK, SK, KHK, JTK.

All authors read and approved the final manuscript.

\section{REFERENCES}

1. Tonnessen BH. Iatrogenic injury from vascular access and endovascular procedures. Perspect Vasc Surg Endovasc Ther 2011;23:128-35.

2. Giswold ME, Landry GJ, Taylor LM, Moneta GL. Iatrogenic arterial injury is an increasingly important cause of arterial trauma. Am J Surg 2004;187:590-2.

3. Rudstrom H, Bergqvist D, Bjorck M. Iatrogenic vascular injuries with lethal outcome. World J Surg 2013;37:1981-7.

4. Yoo TK, Min SK, Ahn S, et al. Major vascular injury during nonvascular surgeries. Ann Vasc Surg 2012;26:825-32.

5. Mills JL, Wiedeman JE, Robison JG, Hallett JW Jr. Minimizing mortality and morbidity from iatrogenic arterial injuries: the need for early recognition and prompt repair. J Vasc Surg 1986;4:22-7.

6. Martin MJ, Perez-Alonso AJ, Asensio JA. Vascular complications and special problems in vascular trauma. Eur J Trauma Emerg Surg 2013;39:569-89.

7. Liu JL, Li JY, Jiang P, et al. Literature review of peripheral vascular trauma: is the era of intervention coming. Chin J Traumatol 2020;23:5-9.

8. Galyfos G, Kerasidis S, Stefanidis G, et al. Iatrogenic and non-iatrogenic arterial injuries in an urban level I trauma center in Greece. Int Angiol 2016;35:526-30.

9. Rudstrom H, Bergqvist D, Ogren M, Bjorck M. Iatrogenic vascular injuries in Sweden: a nationwide study 1987-2005. Eur J Vasc Endovasc Surg 2008;35:131-8.

10. De'Ath HD, Galland RB. Iatrogenic and non-iatrogenic vascular trauma in a district general hospital: a 21-year review. World J Surg 2010;34:2363-7.

11. DuBose JJ, Rajani R, Gilani R, et al. Endovascular management of axillo-subclavian arterial injury: a review of published experience. Injury 2012;43:1785-92.

12. Smolevitz J, Daab L, Liem T, Politano A. Hybrid repair of an iatrogenic left subclavian artery injury: a case report. Ann Vasc Surg 2020;67:563.e7-11. 